\title{
Addition of Metanx in pregabalin partial responders for painful diabetic neuropathy
}

\author{
Allen Mark Jacobs ${ }^{1^{*}}$, Dunlei Cheng ${ }^{2}$ \\ ${ }^{1}$ Saint Louis University School of Medicine, Saint Louis, USA; * Corresponding Author: drjacobs0902@,sbcglobal.net \\ ${ }^{2}$ University of Texas School of Public Health, Dallas, USA
}

Received 20 May 2013; revised 22 June 2013; accepted 30 June 2013

Copyright (C) 2013 Allen Mark Jacobs, Dunlei Cheng. This is an open access article distributed under the Creative Commons Attribution License, which permits unrestricted use, distribution, and reproduction in any medium, provided the original work is properly cited.

\begin{abstract}
Objective: To study the effect of addition of Metanx on burning parasthesias in patients with symptomatic diabetic neuropathy who had obtained only partial symptom resolution with pregabalin. Research design and methods: This was an open-label pilot study. There were 16 patients ( 7 males, 9 females) in the study group and 8 patients ( 3 males, 5 females) in the control group. A patient numeric rating scale $(0-10)$ of neuropathy associated pain was obtained at baseline and at 20 weeks. The control group continued on a fixed dosage of pregabalin without additional medication, while the study group was supplemented with Metanx (a proprietary blend of bioactive B-vitamins), in addition to being continued on a fixed dosage of pregabalin. Results: After 20 weeks, patients in the study group experienced greater pain relief compared to the control group, $(87.5 \%$ vs. $25 \%$ respectively, $p=0.005)$. The average pain score reduction after 20 weeks in the study group was $3 \mathrm{com}$ pared to 0.25 in the control group $(p<0.001)$. Conclusion: Our study suggested that addition of Metanx may be used for the relief of pain in patients that have obtained only partial resolution of symptomatology from pregabalin.
\end{abstract}

Keywords: L-Methylfolate; Methylcobalamin; Metanx; Diabetic Neuropathy

\section{INTRODUCTION}

Diabetic peripheral neuropathy is a complication frequently seen in patients with type 2 diabetes mellitus (DM) [1]. The prevalence of painful peripheral diabetic neuropathy has been reported as $10 \%-20 \%$, with these subjects requiring treatment for painful symptoms [2].

Pregabalin, an alpha 2-delta ligand with analgesic properties, is a frequently used medication which relieves the symptoms of painful diabetic neuropathy. Responders to pregabalin for the management of painful diabetic peripheral neuropathy, defined as greater than $50 \%$ resolution of pain, represent about $48 \%$ of those taking the medication at a $100 \mathrm{mg}$ three times daily dosage [3]. Although a variety of analgesics, antiepileptic and antidepressant medications are available for the treatment of painful diabetic neuropathy, many patients suffering from pain and sleep disturbance achieve only $30 \%-50 \%$ relief of symptoms using a single medication [4]. Increasing the dosage of pregabalin beyond 300 $\mathrm{mg} /$ day is associated with a $70 \%$ improvement in symptom reduction but is associated with an increased incidence of side effects such as dizziness, edema, and somnolence [3,5]. Improvement in the painful symptoms associated with diabetic peripheral neuropathy has also been reported with the use of methylcobalamin $[6,7]$, benfotiamine [6,8], and Metanx [8,9]. Unlike commonly used agents such as pregabalin which are palliative in action, preparations such as L-methylfolate, methylcobalamin or benfotiamine may have a neurotrophic effect on peripheral nerve health and function directly $[6,10,11]$. The purpose of this pilot study was to test the hypothesis that the addition of a neurotrophic agent containing L-methylfolate $3 \mathrm{mg}$, methylcobalamin $2 \mathrm{mg}$, and pyridoxal 5-phosphate $35 \mathrm{mg}$ would increase pain relief when given to subjects using pregabalin for the treatment of painful diabetic peripheral neuropathy.

\section{RESEARCH DESIGN AND METHODS}

This prospective, non-randomized open-label study evaluated the effect of adding Metanx (Pamlab: United States) to existing pregabalin therapy compared to pre- 
gabalin alone in "partial responders" to pregabalin for painful diabetic peripheral neuropathy. A "partial responder" was defined as an individual receiving pregabalin for longer than 4 months with only partial resolution of burning parasthesias of the feet. All study patients had been previously diagnosed with burning parasthesias of the feet secondary to diabetes mellitus, and the diagnosis of diabetic neuropathy was made by history and physical examination. Eligible "partial responders" to pregabalin for the management of symptomatic burning paresthesias were enrolled in the study from an outpatient clinic. Patients participating in the study did not receive any other analgesic preparations. During the study period of 20 weeks, if there was any change in medication regimen, the patients were excluded from the study. In the study group, patients were placed on Metanx two tablets daily in addition to their pregabalin. The control group consisted of 8 "partial responders" who continued on pregabalin only, without any change in the dose of pregabalin during the study period. A patient numeric rating scale $(0-10)$ was used to assess and document neuropathic pain from each participant at the beginning of the study ( 0 weeks) and then again 20 weeks after the patients had been in the study. A rating of 0 indicated no disturbing paresthesias of the feet, and a rating of 10 indicated severe, excruciating burning pain. The primary outcome was the difference between pre- and post-study pain score (numeric rating scale), which represented the severity of neuropathic pain. The proportion of subjects whose pain scores were reduced in each group at the end of the study was compared as well.

\section{Statistical Analysis}

For a continuous target variable, a two-sample t-test was used. For a categorical target variable, Fisher's exact test was used. Type I error rate was set as 0.05 for this study.

Statistical analyses were performed using software SAS 9.1.

\section{RESULTS}

Initially, there were 23 patients in the study group; however, 7 of these patients were withdrawn due to changes in medications. Sixteen patients in the study arm and eight patients in the control arm completed the 20 week trial. None of the patients withdrew from the study due to adverse reaction or intolerability. There were no significant differences at baseline between the two groups in pain score, weight, symptom duration, or hemoglobin A1C (Table 1). There was no significant difference in the dose of pregabalin that patients in both the groups were taking (Fisher's exact test, $\mathrm{P}=0.25$ ). Patients taking pregabalin $100 \mathrm{mg}$ three times a day orally represented $62.5 \%$ and $62.5 \%$ of the patients in the study group and the control group, respectively. Patients on pregabalin $75 \mathrm{mg}$ three times a day orally were $12.5 \%$ and $37.5 \%$ in the study group and the control group, respectively. Finally, $25 \%$ of the patients in the study group were on pregabalin $75 \mathrm{mg}$ twice a day orally, while none of the patients in the control group were on this daily dose. Of the 16 patients in the study group, 14 $(87.5 \%)$ reported resolution of burning paresthesias, whereas only $2(25 \%)$ patients reported a decrease in symptoms in the control cohort [Fisher's exact test $\mathrm{P}=$ $0.005]$. In the study group, the mean pain score at baseline was 3.94, and in the control group, the mean baseline pain score was 3.63 . After the 20 -week study period, patients in the study group had a mean pain score of 0.94 whereas the patients in the control group had a mean score of 3.38. The average absolute pain reduction after 20 weeks in the study group was 3.0 compared to 0.25 in the control group, (two sample t-test, $p<0.001$. Figure 1).

\section{DISCUSSION}

The treatment of symptomatic painful diabetic peripheral neuropathy begins with the optimization of glycemic control [12]. Palliative therapy frequently used for symptomatic control include antiepileptic medications $[13,14]$, tricyclic antidepressants $[15,16]$, selective serotonin norepinephrine reuptake inhibitors [17,18], and opioid analgesics $[19,20]$. Complimentary to adequate glycemic control and analgesic management of painful symptoms, therapy directed at targeting reversal of potential etiologic factors, or neurotrophic therapy, may provide additional relief. The use of the antioxidant alpha lipoic acid has been demonstrated to be effective in reducing symptoms such as stabbing and burning pain, and paresthesias of diabetic neuropathy [21]. Some other compounds that provide relief of symptomatic diabetic neuropathy without the potential side effects are acetyl-L-carnitine, me-

Table 1. Comparison of the baseline characteristics and the pain score between the study and the control group.

\begin{tabular}{ccccc}
\hline Variable & Study Group $^{*}$ & Control Group & Difference $^{* * * *}$ & p-value \\
\hline Weight & 217.94 & 197.63 & 20.31 & 0.205 \\
$\begin{array}{c}\text { Symptom } \\
\text { Duration }\end{array}$ & 15.4 & 13.9 & 1.5 & 0.534 \\
$\begin{array}{c}\text { Hemoglobin } \\
\text { A1c }\end{array}$ & 8.07 & 8.40 & -0.33 & 0.537 \\
$\begin{array}{c}\text { Baseline } \\
\text { Pain Score }\end{array}$ & 3.94 & 3.63 & 0.31 & 0.486 \\
\hline
\end{tabular}




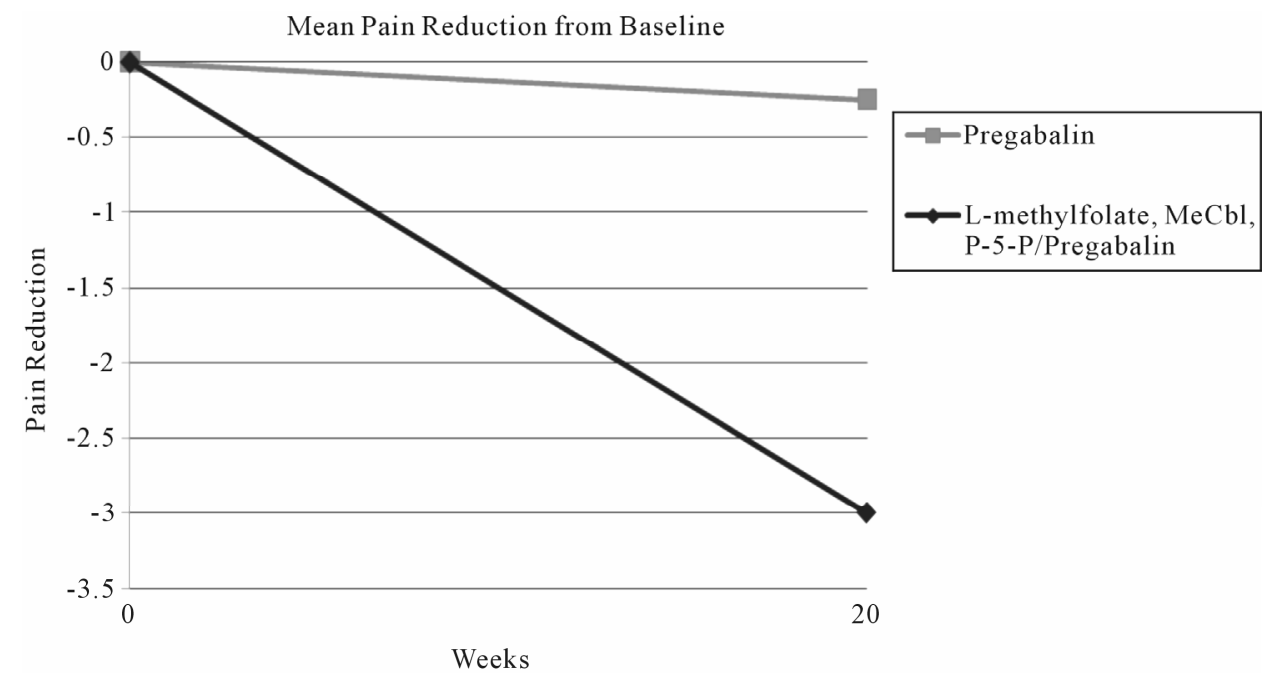

Figure 1. Average pain scores at the baseline and 20th week for both study and control groups.

thylcobalamin, L-methylfolate, and pyridoxine [10]. Methylcobalamin, the neurologically active form of B12, has similarly been employed for the treatment of peripheral neuropathy both as monotherapy and more effectively in combination with other B vitamins [11]. The importance of deficiencies of micronutrients such as folate, B6, B12, and thiamine has been suggested in diabetic neuropathy [22]. Our study results were consistent with a previously published report [23] suggesting that while pregabalin is effective in reducing the frequency and intensity of symptoms associated with diabetic peripheral neuropathy, $50 \%$ of patients utilizing pregabalin continue to experience symptomatology. In the present study, the addition of Metanx to pregabalin improved the painful symptoms of patients in the study group. The effects of Metanx in relief of neuropathic symptoms in the patient with diabetes mellitus may be related to the ability of these agents to lower homocysteine levels and elevate nitric oxide levels. The significance of elevated homocysteine levels in the patient with diabetic neuropathy has been described [24,25]. Because homocysteine is cleared by the kidneys, decreased renal function in patients with diabetes could account for some observed increase in homocysteine levels in such patients $[26,27]$ with resultant peripheral neuropathy. The relationship of total homocysteine and B vitamins, and the ability of B vitamins to reduce homocysteine levels has been elucidated $[28,29]$. In addition, Metformin-related B12 deficiency with resultant increased severity of symptomatic diabetic neuropathy has been described [30]. The ability of methylcobalamin to protect against glutamate induced neurotoxicity in vitro [31], enhance myelin node genesis and nerve regeneration [32], and to reduce symptoms of diabetic neuropathy [7] has been demonstrated in previous studies. The property of folate to effectively metabolize elevated homocysteine levels has also similarly been described [33]. The ability of L-methylfolate to improve nitric oxide synthesis and endothelial function in patients with diabetes may also contribute to symptomatic relief by improvement of endoneurial blood flow [34]. The combination of methylcobalamin and folic acid has been described for the treatment of neuropathy [35]. Finally, pyridoxine deficiency has also been implicated in symptomatic diabetic neuropathy [36]. Our findings are consistent with previously published studies suggesting that patients may receive substantial but only partial relief of symptoms of painful diabetic neuropathy with medications such as pregabalin $[3,4,23]$. The addition of Metanx provides an additional approach to the management of diabetic neuropathy, acting synergistically with palliative agents such as pregabalin, duloxetine, or amitriptyline. Our study results are limited by the small sample size and non-randomized, open-label design. The agents traditionally utilized in the treatment of painful diabetic neuropathy address resolution of symptoms. From our study results, we can speculate that an agent such as Metanx may act in a neurotrophic manner, lowering homocysteine levels, thereby raising neural tissue nitric oxide levels, and improving nerve function and health.

\section{CONCLUSION}

The addition of Metanx, twice daily, reduces the symptoms of diabetic peripheral neuropathy in partial responders to pregabalin. No increased incidence of adverse drug reaction was noted in our study population. Our open-labeled pilot study suggests that the combination of these two agents provides a multimodal approach to the management of diabetic neuropathy, providing the patient with both analgesic and as well as neurotrophic 
therapy. However, a larger randomized controlled trial is needed to confirm these findings.

\section{ACKNOWLEDGEMENTS}

We would like to thank Dr. Tina K. Thethi for editorial assistance with the manuscript. Dr. Jacobs is on the Pamlab speaker bureau but received no funding for the study or manuscript.

\section{REFERENCES}

[1] Boulton, A.J., Vinik, A.I., Arezzo, J.C., et al. (2005) Diabetic neuropathies: A statement by the American Diabetes Association. Diabetes Care, 28, 956-962. doi:10.2337/diacare.28.4.956

[2] Boulton, A.J., Vileikyte, L., Ragnarson-Tennvall, G. and Apelqvist, J. (2005) The global burden of diabetic foot disease. Lancet, 366, 1719-1724. doi:10.1016/S0140-6736(05)67698-2

[3] Lesser, H., Sharma, U., LaMoreaux, L. and Poole, R.M. (2004) Pregabalin relieves symptoms of painful diabetic neuropathy: A randomized controlled trial. Neurology, 63, 2104-2110. doi:10.1212/01.WNL.0000145767.36287.A1

[4] Argoff, C.E., Backonja, M.M., Belgrade, M.J., et al. (2006) Consensus guidelines: Treatment planning and options. Diabetic peripheral neuropathic pain. Mayo Clinic Proceedings, 81, S12-S25. doi:10.1016/S0025-6196(11)61474-2

[5] Richter, R.W., Portenoy, R., Sharma, U., LaMoreaux, L., Bockbrader, H. and Knapp, L.E. (2005) Relief of painful diabetic peripheral neuropathy with pregabalin: A randomized, placebocontrolled trial. Journal of Pain, 6, 253260. doi:10.1016/j.jpain.2004.12.007

[6] Sadekov, R.A., Danilov, A.B. and Vein, A.M. (1999) Diabetic polyneuropathy treatment with Benfotimine. Arzneimittelforschung, 49, 220-224.

[7] Yaqub, B.A., Siddique, A. and Sulimani, R. (1992) Effects of methylcobalamin on diabetic neuropathy. Clinical Neurology and Neurosurgery, 94, 105-111. doi:10.1016/0303-8467(92)90066-C

[8] Walker, M. and Morris, L. (2007) Increased cutaneous sensibility in patients with diabetic neuropathy utilizing a pharmacological approach-clinical case evidence. Vascular Disease Management, 2, 1-8.

[9] Walker Jr., M.J., Morris, L.M. and Cheng, D. (2010) Improvement of cutaneous sensitivity in diabetic peripheral neuropathy with combination L-methylfolate, methylcobalamin, and pyridoxal 5'-phosphate. Reviews in Neurological Diseases, 7, 132-139.

[10] Head, K.A. (2006) Peripheral neuropathy: Pathogenic mechanisms and alternative therapies. Alternative Medicine Review, 11, 294-329.

[11] Zhang, Y.F. and Ning, G. (2008) Mecobalamin. Expert Opinion on Investigational Drugs, 17, 953-964. doi:10.1517/13543784.17.6.953

[12] American Diabetes Association (2008) Standards of medical care in diabetes-2008. Diabetes Care, 31, S12-

\section{S54. doi: $10.2337 / \mathrm{dc} 08-\mathrm{S} 012$}

[13] Backonja, M., Beydoun, A., Edwards, K.R., et al. (1998) Gabapentin for the symptomatic treatment of painful neuropathy in patients with diabetes mellitus: A randomized controlled trial. Journal of the American Medical Association, 280, 1831-1836. doi:10.1001/jama.280.21.1831

[14] Serpell, M.G. (2002) Gabapentin in neuropathic pain syndromes: A randomised, double-blind, placebo-controlled trial. Pain, 99, 557-566. doi:10.1016/S0304-3959(02)00255-5

[15] Duby, J.J., Campbell, R.K., Setter, S.M., White, J.R. and Rasmussen, K.A. (2004) Diabetic neuropathy: An intensive review. American Journal of Health-System Pharmacy, 61, 160-173.

[16] McQuay, H.J., Tramer, M., Nye, B.A., Carroll, D., Wiffen, P.J. and Moore, R.A. (1996) A systematic review of antidepressants in neuropathic pain. Pain, 68, 217-227. doi:10.1016/S0304-3959(96)03140-5

[17] Goldstein, D.J., Lu, Y., Detke, M.J., Lee, T.C. and Iyengar, S. (2005) Duloxetine vs. placebo in patients with painful diabetic neuropathy. Pain, 116, 109-118. doi:10.1016/j.pain.2005.03.029

[18] Sindrup, S.H., Bach, F.W., Madsen, C., Gram, L.F. and Jensen, T.S. (2003) Venlafaxine versus imipramine in painful polyneuropathy: A randomized, controlled trial. Neurology, 60, 1284-1289. doi:10.1212/01.WNL.0000058749.49264.BD

[19] Gimbel, J.S., Richards, P. and Portenoy, R.K. (2003) Controlled-release oxycodone for pain in diabetic neuropathy: a randomized controlled trial. Neurology, 60, 927-934. doi:10.1212/01.WNL.0000057720.36503.2C

[20] Sindrup, S.H., Andersen, G., Madsen, C., Smith, T., Brosen, K. and Jensen, T.S. (1999) Tramadol relieves pain and allodynia in polyneuropathy: A randomised, double-blind, controlled trial. Pain, 83, 85-90. doi:10.1016/S0304-3959(99)00079-2

[21] Ziegler, D., Ametov, A., Barinov, A., Dyck, P.J., Gurieva, I., Low, P.A., et al. (2006) Oral treatment with alpha-lipoic acid improves symptomatic diabetic polyneuropathy: The Sydney 2 trial. Diabetes Care, 29, 2365-2370. doi:10.2337/dc06-1216

[22] Bramswig, S. and Pietrzik, K. (2005) The relevance of selected vitamins (B-vitamins, vitamin C, vitamin E) in patients with type 2-diabetes. Aktuelle Ernährungsmedizin, 30, 311-322. doi:10.1055/s-2005-915301

[23] Rosenstock, J., Tuchman, M., LaMoreaux, L. and Sharma, U. (2004) Pregabalin for the treatment of painful diabetic peripheral neuropathy: A double-blind, placebo-controlled trial. Pain, 110, 628-638. doi:10.1016/j.pain.2004.05.001

[24] Ambrosch, A., Dierkes, J., Lobmann, R., Kuhne, W., Konig, W., Luley, C., et al. (2001) Relation between homocysteinaemia and diabetic neuropathy in patients with Type 2 diabetes mellitus. Diabetic Medicine, 18, 185-192. doi:10.1046/j.1464-5491.2001.00445.x

[25] de Luis, D.A., Fernandez, N., Arranz, M.L., Aller, R., Izaola, O. and Romero, E. (2005) Total homocysteine lev- 
els relation with chronic complications of diabetes, body composition, and other cardiovascular risk factors in a population of patients with diabetes mellitus type 2 . Journal of Diabetes and Its Complications, 19, 42-46. doi:10.1016/j.jdiacomp.2003.12.003

[26] Guttormsen, A.B., Ueland, P.M., Svarstad, E. and Refsum, H. (1997) Kinetic basis of hyperhomocysteinemia in patients with chronic renal failure. Kidney International, $\mathbf{5 2}$, 495-502. doi:10.1038/ki.1997.359

[27] Stabler, S.P., Estacio, R., Jeffers, B.W., Cohen, J.A., Allen, R.H. and Schrier, R.W. (1999) Total homocysteine is associated with nephropathy in non-insulin-dependent diabetes mellitus. Metabolism, 48, 1096-1101. doi:10.1016/S0026-0495(99)90121-X

[28] Obeid, R., McCaddon, A. and Herrmann, W. (2007) The role of hyperhomocysteinemia and B vitamin deficiency in neurological and psychiatric diseases. Clinical Chemistry and Laboratory Medicine, 45, 1590-1606. doi:10.1515/CCLM.2007.356

[29] Scalabrino, G., Buccellato, F.R., Veber, D. and Mutti, E. (2003) New basis of the neurotrophic action of vitamin B12. Clinical Chemistry and Laboratory Medicine, 41, 14351437. doi:10.1515/CCLM.2003.220

[30] Wile, D.J. and Cory, T. (2010) Association of metformin, elevated homocysteine, and methylmalonic acid levels and clinically worsened diabetic peripheral neuropathy. Diabetes Care, 33, 156-161. doi:10.2337/dc09-0606

[31] Kikuchi, M., Kashii, S., Honda, Y., Tamura, Y., Kaneda,
K. and Akaike, A. (1997) Protective effects of methylcobalamin, a vitamin B12 analog, against glutamate-induced neurotoxicity in retinal cell culture. Investigative Ophthalmology \& Visual Science, 38, 848-854.

[32] Watanabe, T., Kaji, R., Oka, N., Bara, W. and Kimura, J. (1994) Ultra-high dose methylcobalamin promotes nerve regeneration in experimental acrylamide neuropathy. Journal of the Neurological Sciences, 122, 140-143. doi:10.1016/0022-510X(94)90290-9

[33] Lonn, E., Yusuf, S., Arnold, M.J., Sheridan, P., Pogue, J., Micks, M., et al. (2006) Homocysteine lowering with folic acid and $\mathrm{B}$ vitamins in vascular disease. The New England Journal of Medicine, 354, 1567-1577. doi:10.1056/NEJMoa060900

[34] Mangoni, A.A., Sherwood, R.A., Asonganyi, B., Swift, C.G., Thomas, S. and Jackson, S.H. (2005) Short term oral folic acid supplementation enhances endothelial function in patients with type 2 diabetes. American Journal of Hypertension, 18, 220-226. doi:10.1016/j.amjhyper.2004.08.036

[35] Venn, B.J., Green, T.J., Moser, R. and Mann, J.I. (2003) Comparison of the effect of low-dose supplementation with L-5-methyltetrahydrofolate or folic acid on plasma homocysteine: A randomized placebo-controlled study. The American Journal of Clinical Nutrition, 77, 658-662.

[36] Jones, C.L. and Gonzalez, V. (1978) Pyridoxine deficiency: A new factor in diabetic neuropathy. Journal of the American Podiatric Medical Association, 68, 646653. 